\title{
Errata : Proceedings of the International Symposium on Magneto-Fluid Dynamics
}

[Revs. Modern Phys. 32 (October 1960), entire issue]

\begin{tabular}{|c|c|c|c|c|}
\hline Page & $\begin{array}{l}\text { Column } \\
(1 \text { or } 2)\end{array}$ & Line & Instead of: & Should read: \\
\hline 712 & 2 & 3 & energy of hydrogen & energy of a hydrogen \\
\hline 720 & 1 & Eq. (4) & $16 \pi^{3} e^{4} c \cdots$ & $2 e^{4} c \cdots$ \\
\hline 742 & 1 & 35 & $\omega_{1}\left|A_{1}\right|$ & $\omega_{1}\left|A_{1}\right|^{2}$ \\
\hline 742 & 2 & 3 & $\int \frac{d t}{d t} \omega^{\frac{1}{2}} \ldots$ & $\int \frac{d}{d t} \omega^{\frac{1}{2}} \cdots$ \\
\hline 782 & & Top & BERSCHADER & BERSHADER \\
\hline 783 & 2 & next to last & luminous charged-particle & luminous, charged-particle, \\
\hline 808 & 1 & 2 & $\begin{array}{r}\mathbf{\Phi}_{i j}^{(n)}(\boldsymbol{\kappa})=\int \mathbf{B}_{i j}^{(n)}(\mathbf{r}) \exp (-i \boldsymbol{k r}) d \mathbf{r} \\
(n=1,2,3)\end{array}$ & $\begin{array}{r}\boldsymbol{\Phi}_{i j}{ }^{(n)}(\boldsymbol{\kappa})=\frac{1}{(2 \pi)^{3}} \int \mathbf{B}_{i j}{ }^{(n)}(\mathbf{r}) \exp (-i \boldsymbol{k r}) d \mathbf{r} \\
(n=1,2,3)\end{array}$ \\
\hline 823 & 2 & 28 & boundary layer & boundary-layer \\
\hline 825 & 2 & Fig. 3 & $N=21$ & $N=2.1$ \\
\hline 826 & 1 & Eq. (24) & $f_{1}^{\prime \prime}$ & $f^{\prime \prime}\left(\eta_{1}\right)$ \\
\hline 858 & 2 & $\begin{array}{c}5 \\
\text { from below }\end{array}$ & as $|x| \rightarrow \infty$. If, as they & $\begin{array}{l}\text { as }|x| \rightarrow \infty \text {. If, as Professor Sears and Pro } \\
\text { fessor Resler }\end{array}$ \\
\hline 883 & 1 & Eq. (13) & $\partial \nu_{2} / \partial t+\nu_{0} \partial u_{2}^{\prime} / \partial x=0$ & $\partial \nu_{2}^{\prime} / \partial t+\nu_{0} \partial u_{2} / \partial x=0$ \\
\hline 887 & 1 & Eq. (56) & $\lambda_{i}=\frac{c_{1} \alpha_{12}^{*}}{\lambda_{R}}=\frac{\alpha_{12}}{2 m_{1} \nu_{0} \omega} \frac{1}{\lambda_{0}}\left(1 \mp 2 \frac{\omega_{i}^{2}}{\omega^{2}} \frac{1}{B^{\frac{\lambda}{2}}}\right)$ & $\lambda_{i}=\frac{c_{1} \alpha_{12}^{*}}{\lambda_{R}}=\frac{\alpha_{12}}{2 m_{1} \nu_{0}} \frac{\omega}{a_{1}^{2}} \frac{1}{\lambda_{0}}\left(1-2 \frac{\omega_{i}^{2}}{\omega^{2}} \frac{1}{B^{\frac{1}{2}}}\right)$ \\
\hline 887 & 1 & 16 & $\begin{array}{l}\text { with } \alpha_{12} \text {, but decreases } \\
\text { with increase of } \omega .\end{array}$ & with $\alpha_{12}$ and $\omega$. \\
\hline 888 & 2 & 8 & $\omega t / 2$ & $\frac{1}{2} \omega \tau$ \\
\hline 903 & 1 & 9 & A necessary condition & A sufficient condition \\
\hline 905 & 2 & $\begin{array}{c}8 \\
\text { from below }\end{array}$ & of field & a field \\
\hline 905 & 2 & 12 & necessary condition & sufficient condition \\
\hline 905 & 2 & 14 & necessary condition & sufficient condition \\
\hline 905 & 2 & 17 & necessary condition & sufficient condition \\
\hline 905 & 2 & 23 & necessary condition & sufficient condition \\
\hline 910 & 2 & $\begin{array}{c}3 \\
\text { from below }\end{array}$ & R. J. Taylor & R. J. Tayler \\
\hline
\end{tabular}




\begin{tabular}{|c|c|c|c|c|}
\hline Page & $\begin{array}{r}\text { Column } \\
(1 \text { or } 2)\end{array}$ & Line & Instead of: & Should read: \\
\hline 926 & 1 & $\begin{array}{c}13 \\
\text { from below }\end{array}$ & has little & may have less \\
\hline 932 & 1 & $\begin{array}{c}26 \\
\text { from below }\end{array}$ & retarded & is retarded \\
\hline 936 & 1 & 2 & sharply defined unless & sharply defined by the magnetic field unless \\
\hline 936 & 1 & 7 & $n_{\min }=\left(m_{+}^{2} e^{2} / 8 \pi e^{3} M\right) v, \quad(10)$ & $n_{\min }=\left(m_{+}^{2} c^{3} / 8 \pi e^{3} M\right) v$ \\
\hline 942 & 1 & 11 & is the vector & is the unit vector \\
\hline 977 & 2 & Eq. (1) & $\mu \frac{d v}{d k}=\cdots$ & $\mu \frac{d v}{d x}=\cdots$ \\
\hline 977 & 2 & Eq. (2) & $\frac{d T}{d y}=\cdots$ & $\frac{d T}{d x}=\cdots$ \\
\hline 981 & 1 & $\begin{array}{c}2 \\
\text { from below }\end{array}$ & very & vary \\
\hline 993 & 2 & 14 & $d \log \sigma / d A^{2}=\cdots$ & $d \log \rho / d A^{2}=\cdots$ \\
\hline 1008 & 2 & 9 & $(1 \equiv \delta / \delta t)$ & $\left({ }^{\prime} \equiv \partial / \partial t\right)$ \\
\hline 1010 & 2 & 10 & that & than \\
\hline 1010 & 2 & 19 & $\begin{array}{l}\text { moving with a velocity of } \lesssim 10^{7} \mathrm{~cm} / \mathrm{sec} \\
\text { may give rise }\end{array}$ & $\begin{array}{l}\text { moving with a velocity of } \cong 10^{7} \mathrm{~cm} / \mathrm{sec}- \\
\text { may give rise }\end{array}$ \\
\hline 1012 & 2 & 16,17 & $\begin{array}{l}\text {... expresses the number of charged } \\
\text { particles created per unit volume and } \\
\text { time per charged. . . }\end{array}$ & $\begin{array}{l}\ldots \text { expresses the number of charged } \\
\text { particles which are created per unit time } \\
\text { per charged. . . }\end{array}$ \\
\hline 1012 & 2 & footnote 18 & $\begin{array}{l}{ }^{18} \mathrm{~B} \text {. Lehnert, Arkiv Fysik (to be } \\
\text { published). }\end{array}$ & $\begin{array}{l}{ }^{18} \text { B. Lehnert, Arkiv Fysik 17, } 177 \text { (1960); } \\
\text { 18, } 251 \text { (1960). }\end{array}$ \\
\hline 1025 & 1 & 9 & in turn have & are driven by \\
\hline 1025 & 1 & 11 & east to west & north to south \\
\hline
\end{tabular}

